\title{
An analysis of current state of the art software on nanopore metagenomic data
}

\author{
Samantha C Pendleton ${ }^{1 *}$ \\ sap21@aber.ac.uk
}

October 06, 2017

${ }^{1}$ Institute of Mathematics, Physics and Computer Science, Aberystwyth University

Last Updated: March 26, 2018

\begin{abstract}
Context Our insight into DNA is controlled through a process called sequencing. Until recently, it was only possible to sequence DNA into short strings called "reads". Nanopore is a new sequencing technology to produce significantly longer reads. Using nanopore sequencing, a single molecule of DNA can be sequenced without the need for time consuming PCR amplification (polymerase chain reaction is a technique used in molecular biology to amplify a single copy or a few copies of a segment of DNA across several orders of magnitude).

Aims Metagenomics is the study of genetic material recovered from environmental samples. A research team from IBERS (Institute of Biological, Environmental $\&$ Rural Sciences) at Aberystwyth University have sampled metagenomes from a coal mine in South Wales using the Nanopore MinION and given initial taxonomic (classification of organisms) summaries of the contents of the microbial community.

Methods Using various new software aimed for metagenomic data, we are interested to discover how well current bioinformatics software works with the data-set. We will conduct analysis and research into how well these new state of the art software works with this new long read data and try out some recent new developments for such analysis.

Results Most of the software we used worked very well: we gained understanding of the ACGT count and quality of the data. However some software for bioinformatics don't seem to work with nanopore data. Furthermore, we can conclude that low quality nanopore data may actually be quite average.
\end{abstract}

Keywords - bioinformatics, nanopore, metagenomic

\section{Introduction}

A wide variety of software is available today for bioinformatics. Majority of these tools that were created aim for short DNA reads, which researchers are familiar to working with.

Though new sequencing methods have made it possible to work with longer reads, making us curious if the bioinformatics tools also work with these data-sets. These significantly longer reads are going to be analysed with numerous bioinformatics tools; we want to know how the tools work and analyses nanopore data, specifically metagenomic data.

Using different software, we want to analyse the data to observe sequence similarities and find out what bacteria/species that resides within in mine. Our aim is to specifically observe quality, however we will also be looking into reads, ACGT content (in-depth GC analysis), plus time.

From a coal mine located in South Wales, DNA was extracted by a research team from the Institute of Biological, Environmental \& Rural Sciences (IBERS); these mines had no internet access available.

The samples extracted were run in the nanopore technology, results were analysed in a pre-printed paper[4].

Two data sets were concluded from the mine expeditions; both from the same mine. There extracted 4 DNA samples for BP_v1, generated Dec-2016. 8 samples were extracted for BP_v2, generated Apr-2017.

Version 2, BP_v2, is better quality as the researcher had optimised the DNA extraction to improve recovery yields from this kind of environment by using an improved protocol[3].

${ }^{*}$ Institute of Biological, Environmental and Rural Sciences, Aberystwyth University 


\section{Method}

A great deal of in-depth analysis of the data-sets will be conducted and discussed throughout. Whilst in the mine, the samples were run through the new MinION ${ }^{1}$ technology, developed by Oxford Nanopore ${ }^{2}$.

I downloaded source code of various bioinformatics tools and installed them, some were run through the use of the IBERS cluster server - looking into sequence similarity of the FAST5 ${ }^{3}$ data, with the tools: poretools, Goldilocks, FastQC and BLAST.

\section{1 poretools}

poretools[7] is a toolkit for analysing nanopore sequence data - it was developed in Aug-2014 with 77 issues as of Sep-2017 on Github ${ }^{4}$ - possibly explaining potential errors and bugs.

\subsubsection{Read Lengths}

BP_v1 had more short reads, with a total of 1,770 files. Whilst BP_v2 generated consistently longer reads and a total of 3,019 files - it was not quality filtered; it had MUX genes, which were later removed for analysis. In the histograms, we can see the comparison of the data-sets; they were recreated from the ones used in the IBERS pre-print paper[4].

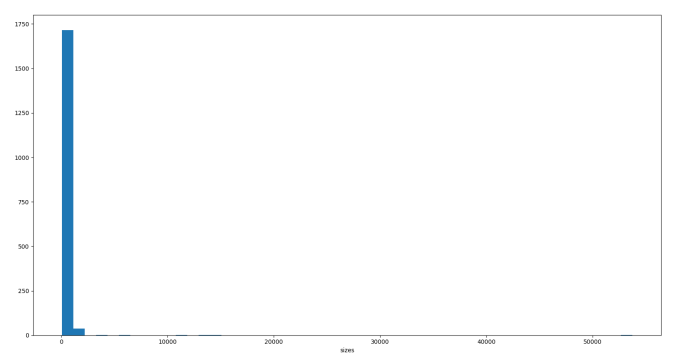

Figure 1: Histogram of BP_v1:- $x$-axis: read length (size); y-axis: cumulative frequency (count).

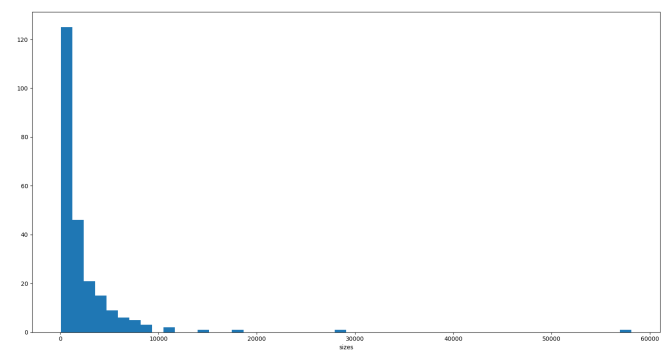

Figure 2: Histogram of BP_v2:- $\mathrm{x}$-axis: read length (size); y-axis: cumulative frequency (count).
After some quality tests, it was observed that the longest reads were low in quality and so most likely errors whilst the samples were running through the MinION - we limited the data to 10,000 base pairs (bp) and compared the full data-set to the limited set frequently throughout.

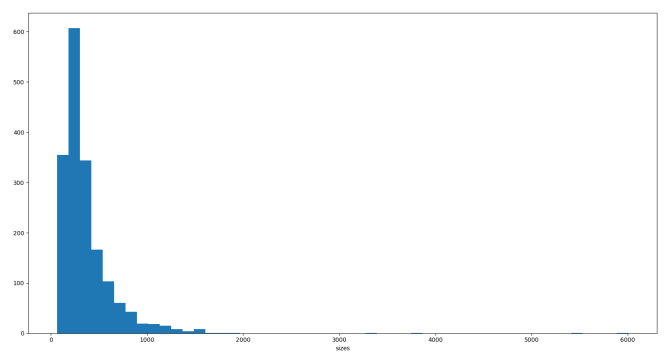

Figure 3: BP_v1 limited to 10,000 bp:- $x$-axis: read length (size); y-axis: cumulative frequency (count).

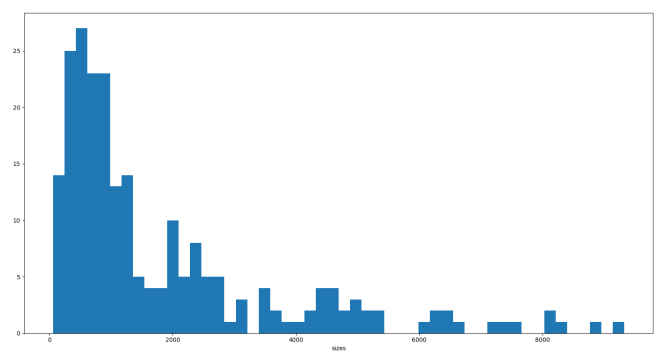

Figure 4: BP_v2 limited to 10,000 bp:- $x$-axis: read length (size); y-axis: cumulative frequency (count).

\subsubsection{Quality}

The quality of both data-sets averaged low with BP_v2 being slightly higher in quality compared to BP_v1. poretools' qualpos function produces box plots, these plots are useful displaying results of low quality, however, there's an unusual number of plots scoring at 60: these are outliers which could be a result due to software not updated (as stated earlier the high number of issues on the Git repository).

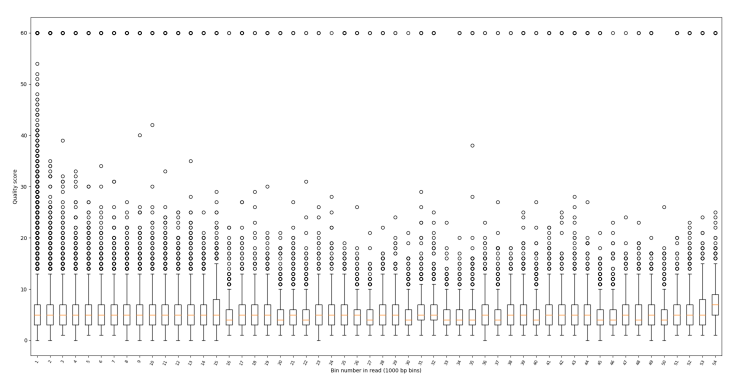

Figure 5: poretools' use of qualpos of BP_v1:- $x$-axis: bin number in read; y-axis: quality score.

\footnotetext{
${ }^{1}$ MinION, "the only portable real-time device for DNA and RNA sequencing." https://nanoporetech.com/products/minion

${ }^{2}$ Oxford Nanopore, "enable the analysis of any living thing, by any person, in any environment". https://nanoporetech.com

${ }^{3}$ FAST5, "file-type in bioinformatics from from Oxford Nanopore". http://bioinformatics.cvr.ac.uk/blog/exploring-the-fast5-format/

${ }^{4}$ poretools, "a toolkit for working with nanopore sequencing data from Oxford Nanopore". https://github.com/arq5x/poretools
} 


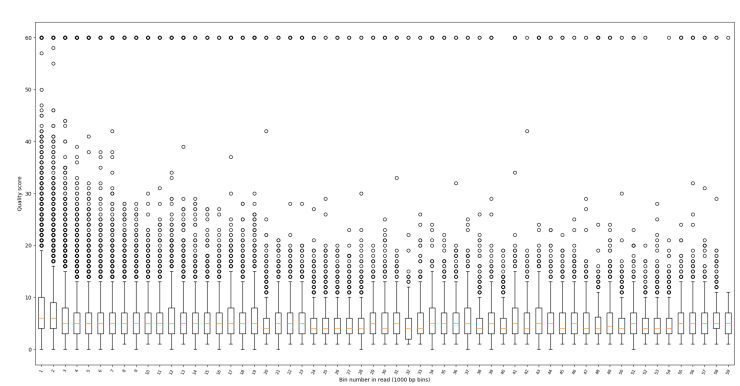

Figure 6: poretools' use of qualpos of BP_v2:- x-axis: bin number in read; y-axis: quality score.

Using poretools' qualdist (summary quality scores) function, we can observe the quality count: we can analyse the lack of count for letters: concluding further that both data-sets are poor.

Note: letters are what we are aiming for, these are good quality scores; ' $\%$ ' is a specific symbol that relates to bad quality - both data sets are high in this symbol; count of 116,956 in BP_v1 \& 81,437 in BP_v2. We can see that BP_v1 has a higher count of 'bad' symbols giving more evidence that BP_v1 is lower quality than BP_v2.

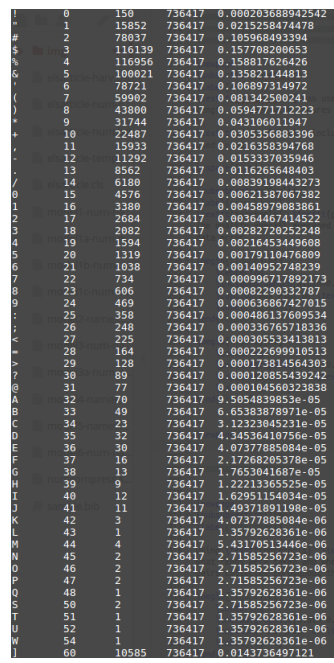

Figure 7: poretools' qualdist of BP_v1.

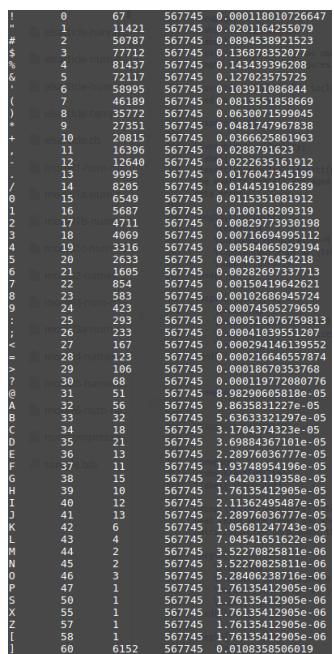

Figure 8: poretools' qualdist of BP_v2.

\subsubsection{Time}

The research team left the mine at 50 minutes - BP_v1 was paused for 6 hours then continued at a researcher's home. Meanwhile BP_v2 ran throughout the return journey: DNA reads were affected after they left the mine during the elevator trip back to the surface plus the car journey (breaks, bumps, and going up/down a hill).

In the plots below, there are sections that show large vertical jumps, which are sudden production of base pairs: after studying, these are sections which were high in AT repetitive reads.

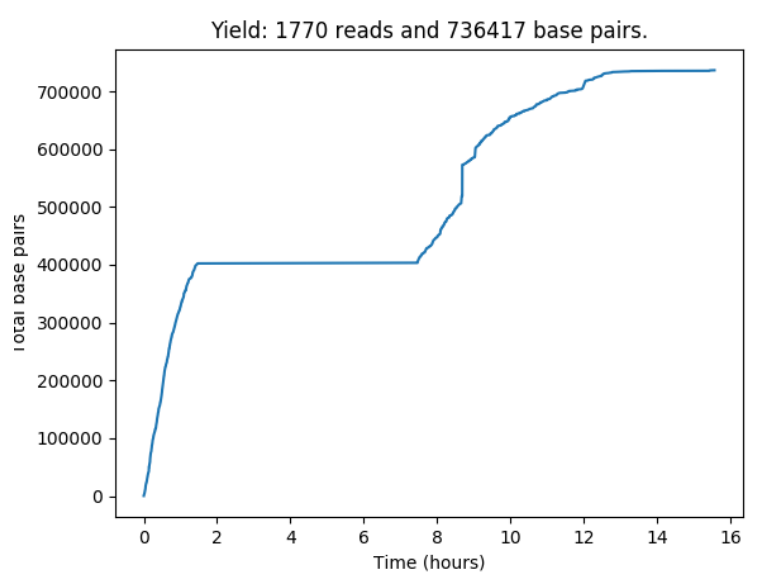

Figure 9: Time graph of BP_v1 using yield_plot:$\mathrm{x}$-axis: time (hours); $\mathrm{y}$-axis: count of total base pairs.

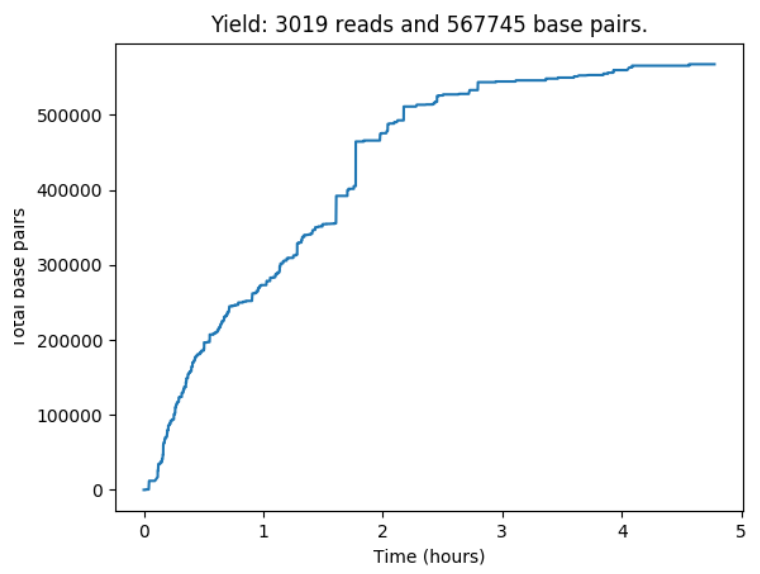

Figure 10: Time graph of BP_v1 using yield_plot:$\mathrm{x}$-axis: time (hours); $\mathrm{y}$-axis: count of total base pairs.

There is an linear increase of base pairs as time increases for both data-sets, as we would expect (ignoring the pause for BP_v1). Both data-sets seem to have collected 400,000 base pairs within two hours BP_v1 collected 736,417 base pairs over 10 hours (16 hours total with a 6 hour pause) and BP_v2 collected 567,745 in 5 hours.

We can see at 9 hours for BP_v1, there was a sudden long peak, reduced/no yield in DNA - this is potentially the longest read of the data set and coincidental A heavy. 
The top 5 longest reads of BP_v2 were taken after 50 minutes - we can bring facts together to conclude why there was data error: after leaving the mine, the team continued to run the analysis during the journey and and car journey affected the sequences.

However, other results from different tests told us how the top $5 \mathrm{~T}$ heavy reads of BP_v2 were not the same as the top 5 longest reads. Moreover, the top $5 \mathrm{~T}$ heavy reads were majority after the first hour too, despite one read that was actually within the 50 minutes; after analysing this read through BLAST, we see that despite high in $\mathrm{T}$, it actually had bacteria and fungus, but also sequence similarities of plant and animal.

\subsection{Goldilocks}

Goldilocks [9] was developed to "quickly locate interesting regions on the human genome that expressed a desired level of variability, which were 'just right' for later variant calling and comparison" - it was developed in Aug-2014 with last update Jul-2016 and 7 issues as of Sep- $2017^{5}$.

The use of Goldilocks allowed us to observe ACGT content and GC in more detail through a scatter plot.

Note: Goldilocks produces linear graphs and the reads are in cardinal order: read as data position in file, which is most likely time.

\subsubsection{GC}

GC in DNA analysis tells us about the stability: DNA with low GC content is less stable.

$\mathrm{A}$ adenine pairs with $\mathrm{T}$ thymine, and $\mathrm{C}$ cytosine pairs with G guanine. Majority of researchers look for higher ratio in GC compared to AT.

BP_v1 is quite scattered with a cluster at location 100,000 - BP_v2 average $0.7 \mathrm{GC}$ content with an unusual section of low GC content at location 300,000.

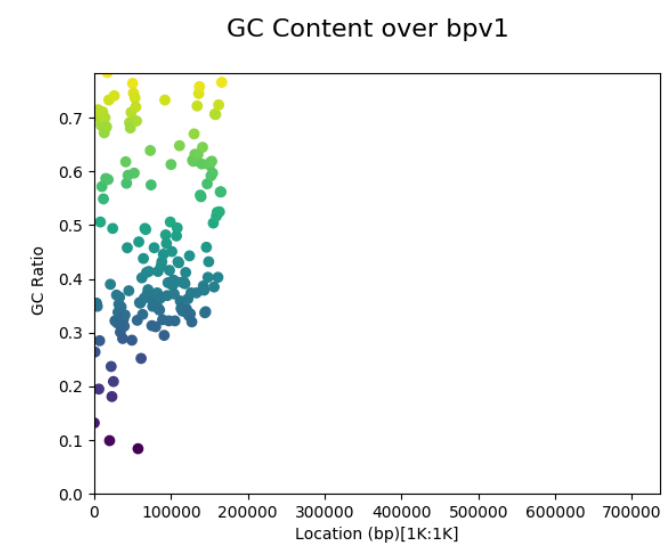

Figure 11: GC ratio of BP_v1:- $\mathrm{x}$-axis: the location along the genome; $y$-axis: the value of the censused region.

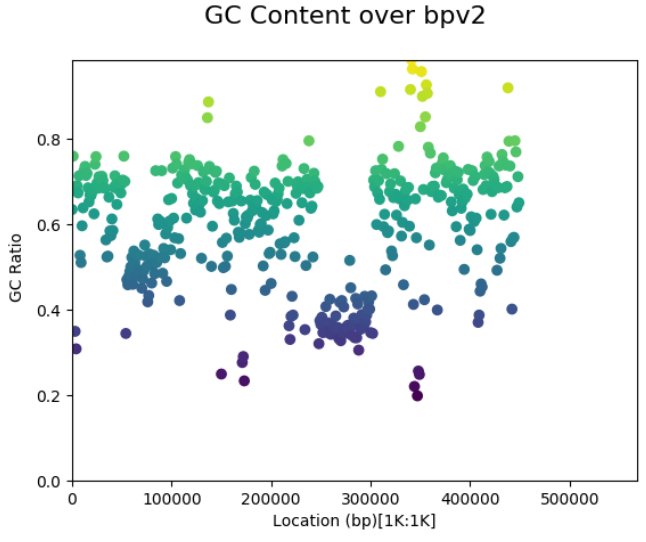

Figure 12: GC ratio of BP_v2:- $\mathrm{x}$-axis: the location along the genome; $y$-axis: the value of the censused region.

\subsubsection{ACGT}

The NucleotideCounterStrategy shows that both data-sets have a high number of Ts with BP_v2 having a peak of $\mathrm{T}$ at base pair location 250000-300000; BP_v1 has an unusual peak of A at location 75000-125000.

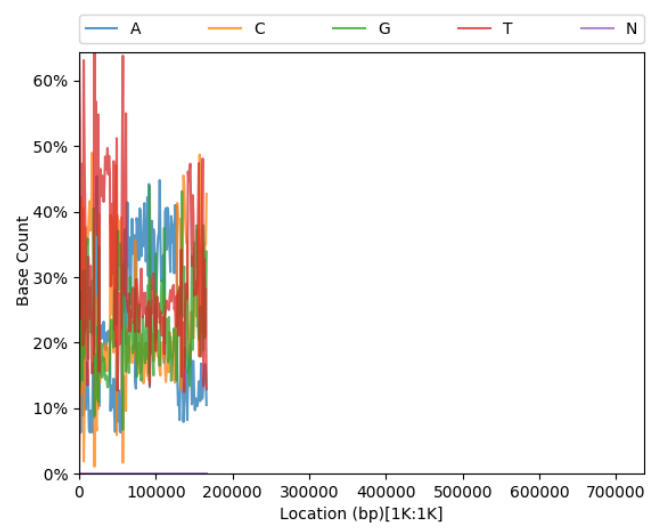

Figure 13: ACGT content of BP_v1:- $\mathrm{x}$-axis: the location along the genome; $y$-axis: percentage of content cover.

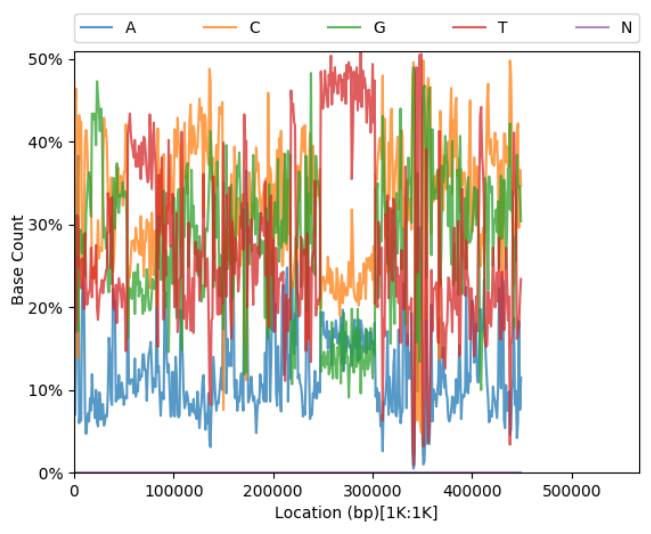

Figure 14: ACGT content of BP_v2:- $\mathrm{x}$-axis: the location along the genome; $y$-axis: percentage of content cover.

Goldilocks's g.query was used to find the reads with the most Ts in BP_v2 \& most As in BP_v1 to which we then used the following command in a Linux terminal:

\footnotetext{
${ }^{5}$ Goldilocks, "locating genetic regions that are just right". https://github.com/SamStudio8/goldilocks
} 
head -n10 BP_v2.fasta.fai

This method is to observe the top longest reads for both data sets, we did a quality check on both: the longest reads were poor in quality (to be explained further).

Note: to use the FAST5 files with Goldilocks, we had to use poretools to convert them to FASTA $^{6}$ SAMtools [6] was used to index FASTA files so we could use them with Goldilocks.

textttporetools was also used to convert the FAST5 files to FASTQ $^{7}$ for use in FastQC.

\subsection{FastQC}

FastQC[1] was released Apr-2010, the oldest bioinformatics tool we are using, but it's most recent updated was Mar-2016. It provides basic statistics of the datasets including read length and quality.

Note: FastQC has produced graphs that are not useful as the $\mathrm{x}$-axis is uniform but stretched; whereas Goldilocks produces linear graphs: we want to observe read positions in linear form. Moreover, FastQC graphs do not display the whole data-sets; they are, unknown to us, limited.

\subsubsection{Read Length}

Some statistical analysis of the data-sets that FastQC provides includes details of read length and GC content. There are 1761 total sequences in BP_v1, whilst BP_v2 has 236 - BP_v1 has more reads since they are shorter, BP_v2 has less in total but they're longer reads.

\subsubsection{GC}

The GC ratio of BP_v1 is $51 \%$, whilst BP_v2 is $60 \%$. Comparing GC in FastQC \& Goldilocks may be quite difficult here due to difference of graphs: FastQC plots are line whilst Goldilocks creates scatter.

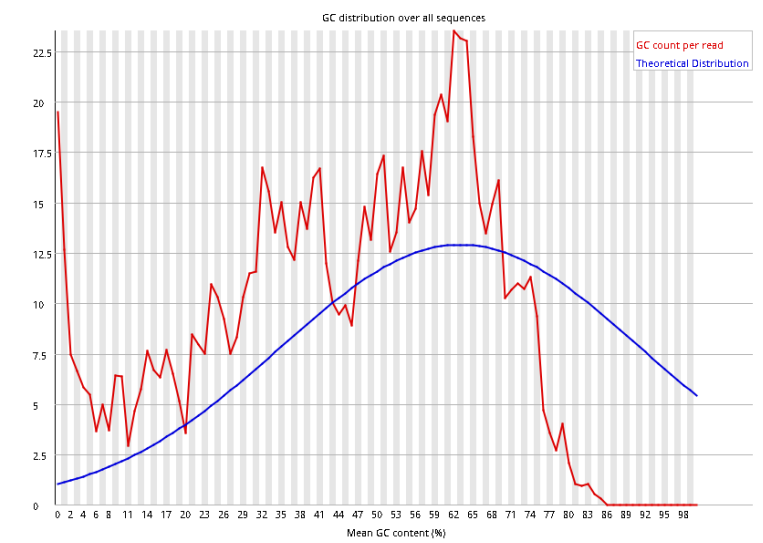

Figure 15: Plot of GC content of BP_v1 in FastQC:$\mathrm{x}$-axis: mean content (\%); $\mathrm{y}$-axis: GC count per read.

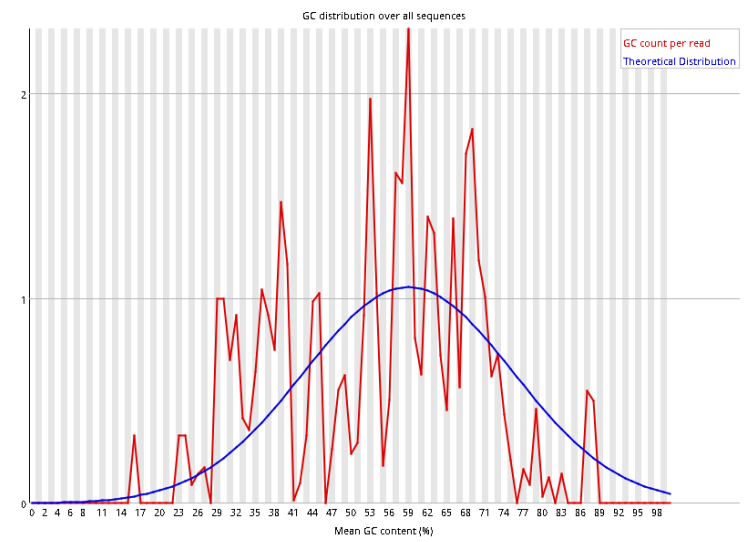

Figure 16: Plot of GC content of BP_v2 in FastQC:$\mathrm{x}$-axis: mean content $(\%)$;-axis: GC count per read.

\subsubsection{ACGT}

Plotting ACGT content with FastQC produces truncated $x$-axis: they scale in an unusual way. Comparing to Goldilocks' ACGT plots, we can see visible differences - T count in BP_v2 steadily increases over time, as if logarithmic. These results of the FastQC plots are due to the truncated $\mathrm{x}$-axis and it's scaling.

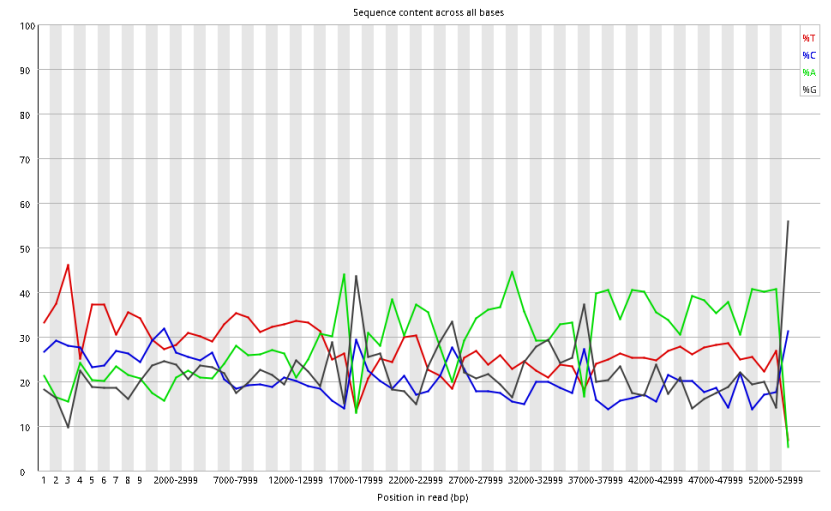

Figure 17: Plot of ACGT sequence content of BP_v1 in FastQC:- $\mathrm{x}$-axis: position in read (bp); $\mathrm{y}$-axis: score.

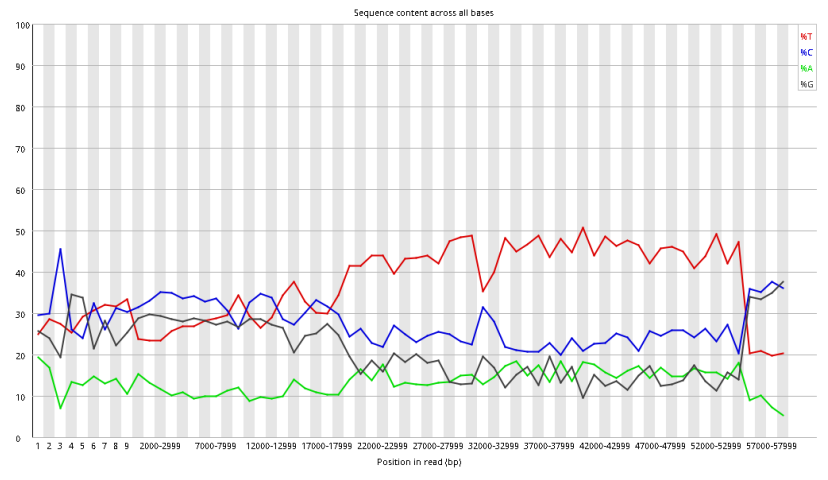

Figure 18: Plot of ACGT sequence content of BP_v2 in FastQC:- $\mathrm{x}$-axis: position in read (bp); y-axis: score.

\begin{tabular}{cccc} 
A & C & G & T \\
\hline green & blue & black & red
\end{tabular}

\footnotetext{
${ }^{6}$ FASTA, "file-type in bioinformatics". https://zhanglab.ccmb.med.umich.edu/FASTA/

${ }^{7}$ FASTQ, "file-type storing the Phred qualities". http://maq.sourceforge.net/fastq.shtml
} 


\subsubsection{Quality}

Quality tests were conducted using FastQC, as we discovered earlier with poretools both BP_v1 and BP_v2 are low in quality. Despite the unusual ACGT plots of FastQC, the quality plots back-up the findings from poretools: despite FastQC's results are limited from the $\mathrm{x}$-axis, there are no outliers unlike poretools.

The test of quality are again displayed as box plots. The below plots are similar to poretools: low quality. However, when extracting some single reads of BP_v2 and plotting, some values plotted in the medium range.

FastQC results are very useful, despite the limited box plots and strected $\mathrm{x}$-axis. As stated earlier, FastQC's x-axis is uniform but stretched, at first were misunderstood as logarithmic graphs; in these instances we prefer Goldilocks' plots due to their graphs being linear.

The plots background colour scheme (observing data subsiding in the red area) is a unique feature.

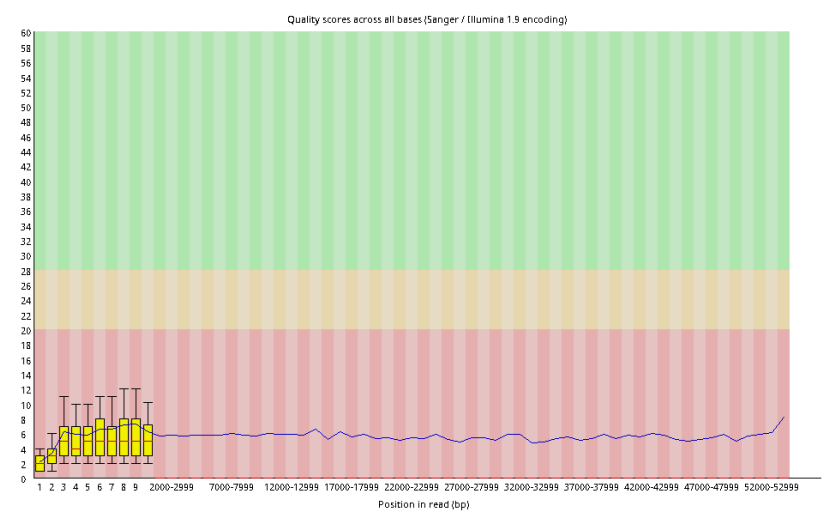

Figure 19: Plot of BP_v1 reads in FastQC quality graph:$\mathrm{x}$-axis: position in read; $\mathrm{y}$-axis: score.

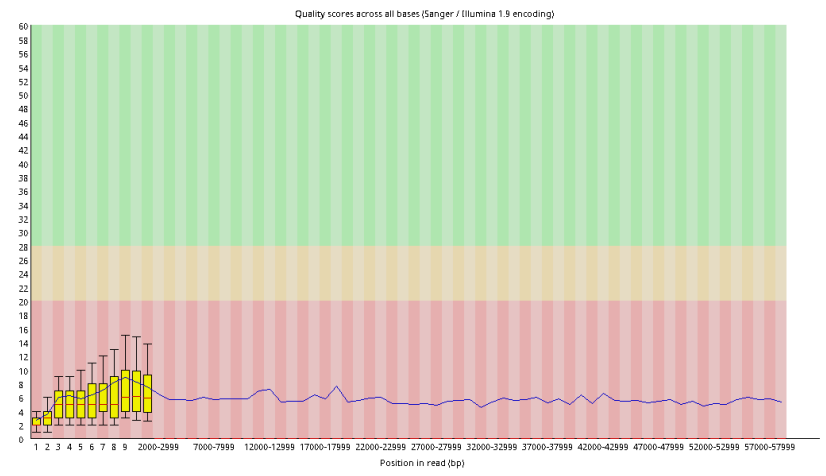

Figure 20: Plot of BP_v2 reads in FastQC quality graph:$\mathrm{x}$-axis: position in read; $\mathrm{y}$-axis: score.

As mentioned earlier, we had two versions of both data-sets - one original of both sets and the other were both sets limited to 10,000 base pairs.
When limiting the data-sets to 10,000 base pairs, we get the following plots below - BP_v2's highest values on the box plots almost reach the medium/decent quality range - in a further study some random reads that were tested in FastQC's quality test, we discovered that many ranged in the medium area - perhaps the data that is reliable was actually a medium score quality but majority of the data is low quality that it lowers the average.

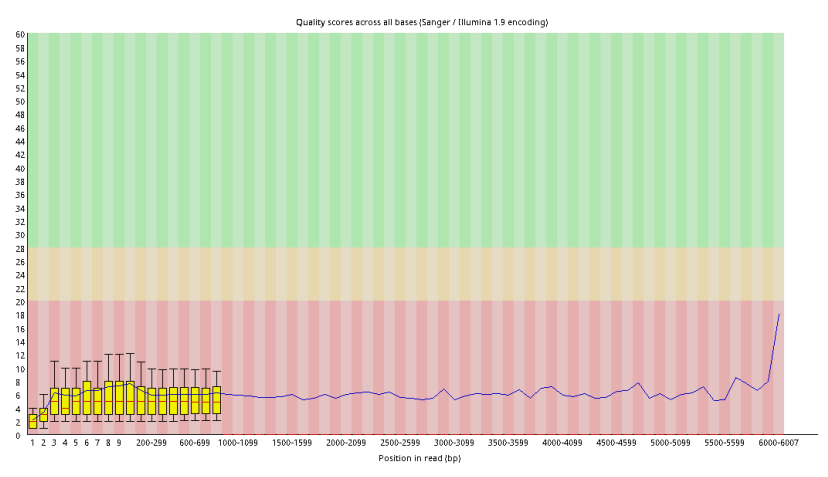

Figure 21: Plot of BP_v1 reads in FastQC quality graph, limited to 10,000 bp:- $\mathrm{x}$-axis: position in read; $\mathrm{y}$-axis: score.

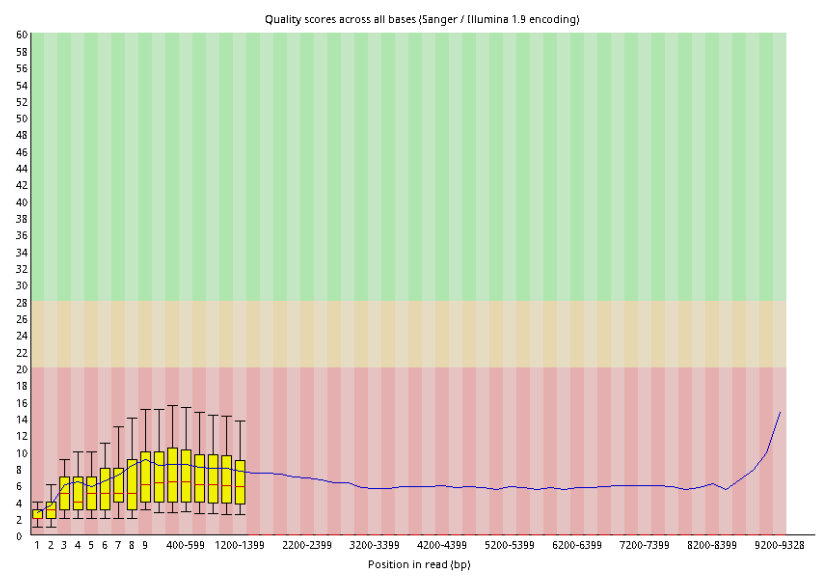

Figure 22: Plot of BP_v2 reads in FastQC quality graph, limited to 10,000 bp:- $\mathrm{x}$-axis: position in read; $\mathrm{y}$-axis: score.

\subsection{BLAST}

BLAST $^{8}$ for Basic Local Alignment Search Tool is an algorithm for comparing sequence information: the nucleotides of DNA sequences. We used this tool for analysing sequence similarities:- alignment lengths: aiming for results within hundreds; bit-score: high bitscores mean better sequence similarity; and percentage of identical matches. We used BLASTn on both data-sets and searched the results (taxon IDs) in the NCBI database.

Note: links in this section may have expired.

\footnotetext{
${ }^{8}$ BLAST, "finding regions of similarity between biological sequences". https://blast.ncbi.nlm.nih.gov/Blast.cgi
} 


\section{Longest reads}

Further tests included using nucleotide BLAST (BLASTn) with discontigious MEGABLAST on the longest reads of both data sets to yet further prove the quality is low: the query cover was $0 \%$ of tiny hits, under 100 base pairs (bp).

BP_v1

Species: Japanese rice fish \& Zebrafish (animals), Fruit flies (insects).

Dictyostelium discoideum: species of soil-living amoeba - commonly referred to as slime mould.

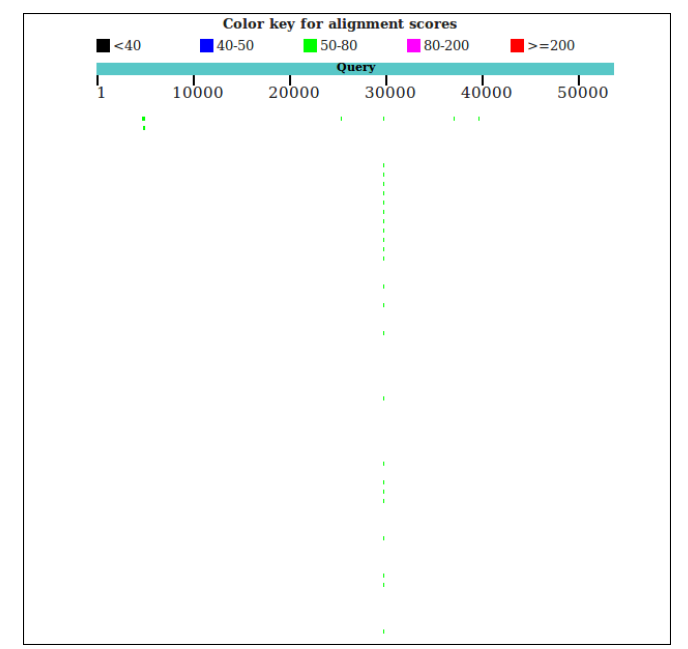

Figure 23: BP_v1 BLAST alignment score of the longest read.

BP_v2

Species: Mouse \& Tapeworm (animals), Tomato \& Kiwi (plant), Fruit flies (insects), Human.

Parasites (including flatworms \& malaria), Fungus

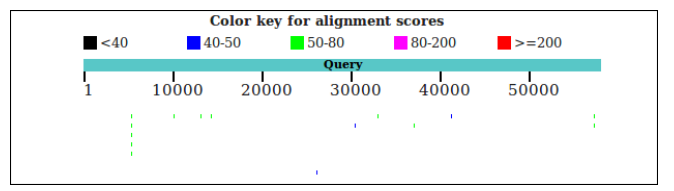

Figure 24: BP_v2 BLAST alignment score of the longest read.

\section{Random reads}

Due to the long reads being low in quality and not many results of reliable species, further tests on the data sets were conducted: specifically we aim to blast random, shorter reads.

After extraction of random numerous shorter reads from BP_v1 and the use of BLAST: no similarities appeared - the reads were mostly: AT content (short and repetitive).

Many random reads of BP_v2 were tested and like results of $\mathrm{BP}_{-} \mathrm{v} 1$, no similarities appeared. However, after extracting a random read from BP_v2 - we can observe this data read was bacteria, Anaerolinea thermophila ${ }^{9}$.
There was a query cover of $17 \%$.

channel_349_bf5a3f39-3e35-4870-82eca7f9679d4000_qscore_9_read_score_-1.8

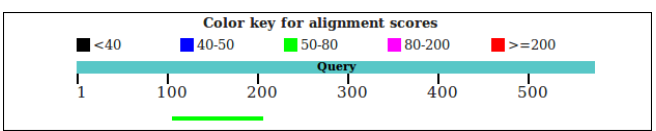

Figure 25: Alignment Score of a random BP_v2 read.

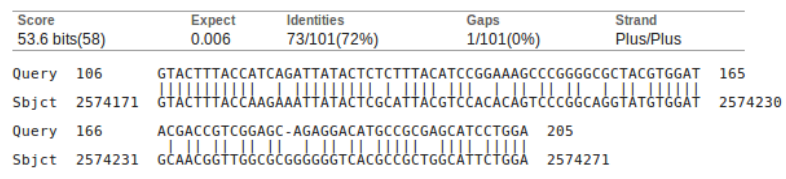

Figure 26: Alignment: Anaerolinea thermophila UNI-1 DNA, complete genome.

Anaerolinea thermophilia is thermophilic bacteria which thrives in high temperatures (41-122 degrees Celsius) - this result is quite unusual as the mine was not significantly at a high temperature; a researcher stated the mine was 15-20 degrees.

For further analysis, we extracted another random read from BP_v2 (ID: 164). Despite no actually good hit, a reoccurring factor was E-coli.

The highest result of E-coli can be seen below: there was a query cover of $1 \%$ commonly.
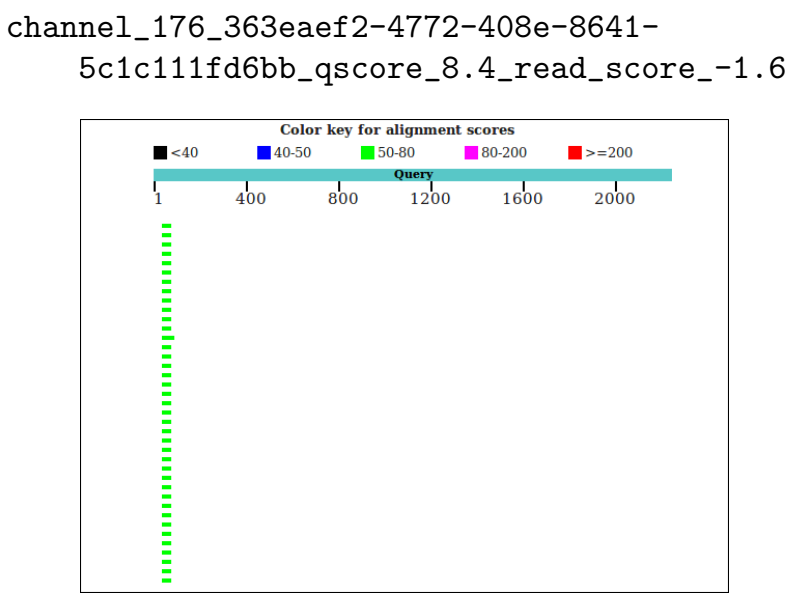

Figure 27: Alignment Score of a random BP_v2 read.

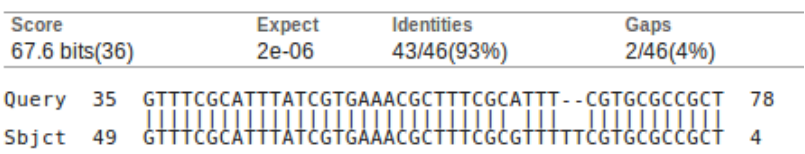

Figure 28: Alignment: Escherichia coli transposon $\mathrm{Mu}$ dl-R insertion site.

Escherichia coli transposon; a transposon is expected, it is a sequence of DNA that can move to new positions within the genome: jumping genes ${ }^{10}$.

Furthermore, the alignment score seems to be 100: these are not genes however could be 10,000 matches of 1,000 genes.

\footnotetext{
${ }^{9}$ Anaerolinea thermophila, NCBI description of filamentous thermophilic bacteria. https://www.ncbi.nlm.nih.gov/pubmed/14657113

${ }^{10}$ Transposons, "The Jumping Genes". https://www.nature.com/scitable/topicpage/transposons-the-jumping-genes-518
} 


\section{Whole data-sets}

Using AU IBERS cluster server, I was able to blast the data-sets as a whole, rather than just blasting long or random reads. We obtained a bunch of taxon IDs that we searched in NCBI database.

This method was used as the NCBI BLAST website would timeout due to the data-sets being large: $\mathrm{CPU}$ usage limit was exceeded. I had to BLAST locally with the nt nucleotide collection/database[2] ( $\mathrm{nr}$ is the proteins collection).

BP_v1 had poor results - unfortunately the highest result of alignment length was 49; highest bit-score was 67.6 ; and mostly $100 \%$ identical matches but this is because the reads are short.

\section{Species found:}

$\triangleright$ Anaeromyxobacter dehalogenans 2CP-C: strain that efficiently reduces metals: ferric iron, $\mathrm{Fe}(\mathrm{III})$, and oxidised uranium (bacteria)

$\triangleright$ Capsicum annuum: Sweet and chili peppers (plant)

$\triangleright$ Pygocentrus nattereri: Red-bellied piranha (animal)

BP_v2 had better results ranging from 300 to 900 in alignment score; bit-scores were as high as 440; and percentage of identical matches varied due to longer reads: for the top 5 highest alignment scores, the average result was $74.941 \%$.

\section{Bacteria found:}

$\triangleright$ Neorhizobium galegae: bacteria that forms nitrogenfixing root nodules

$\triangleright$ Rhodoplanes: a phototrophic genus of bacteria - organisms that carry out photosynthesis

$\triangleright$ Azorhizobium caulinodans: bacteria that forms a nitrogen-fixing symbiosis with plants of the genus Sesbania (flowering plants in pea family)

$\triangleright$ Bordetella flabilis: recovered strains from cystic fibrosis (from human respiratory specimens)

$\triangleright$ Nitrosomonas: bacteria that oxidizes ammonia into nitrite as a metabolic process; found in areas that contains high levels of nitrogen compounds

$\triangleright$ Rhodoplanes: bacteria organisms that carry out photosynthesis

$\triangleright$ Sideroxydans lithotrophicus: bacterium isolated from iron contaminated groundwater

\footnotetext{
${ }^{11}$ Python, Programming Language. https://www.python.org

${ }^{12}$ Matplotlib, Python 2D plotting library. https://matplotlib.org
}

\section{Discussion}

The tools as a whole each had their own advantages; discussing poretools, as it is a software designed for nanopore data, it is handy with lots of features, the histograms plus other plots were very useful: we were able to observe the time plots, read length, and quality information with the data we gathered from the other tools. Though the outliers in the box plots don't agree with the quality visualisations from FastQC so we aren't able to detect whether this is due to poretools' need for updating or something with poretools that we aren't able to figure out.

Goldilocks on the other hand, was not designed for nanopore data however it works very well. If it were updated it could become a very practical tool for this new data. The use of Python ${ }^{11}$ made us able to alter the functions in our own way: Matplotlib ${ }^{12}$ enables us to alter the plots: making them log, changing colours, plus more - altering the ACGT plots with Matplotlib in Python allowed us the alter the colours of GC and observe the AT reads separately.

FastQC offers a variety, the basic statistical analysis it provides on the software is quite a handy observation. However, this bioinformatics tool is not suitable for nanopore data due to graph truncation. When shortening the reads to $10,000 \mathrm{bp}$, the plots seemed to be a little more relaible, however we still cannot understand why there are few box plots with a stretched $\mathrm{x}$-axis.

BLAST is very functional and useful, but slow. BLASTING separate reeds is practical, however if the data is high quality the results would be much more reliable. As our data was low in quality, we had to BLAST random shorter reads, which came back with somewhat reliable results however we cannot continue blasting random reads out of the few thousands of reads in total.

Though despite BLASTING the data-sets as a whole taking quite some time, we were able to gain some good results, especially for BP_v2.

The more we see similarities in results from the different software, the more the can rely on them in future for nanopore data. When new bioinformatics tools are released, we can use the old tools as a comparison.

On the other hand, where there are differences, it is quite difficult to know whether which tool is more accurate. There are also external factors: if one tool has random outliers yet another does not, are these outliers correct or perhaps it's a fault with the software? Plus we need consider the premises that if a software is faster than another, is it more reliable or precise? 


\section{Conclusions}

I had aimed to demonstrate more software, however the BLAST jobs alone took many hours and another piece of software, Centrifuge[5], couldn't run as it didn't have enough RAM on the IBERS compute cluster to scale to the input data.

Centrifuge would have been a great bioinformatics tool to use as it is a tool for for metagenomic sequences ${ }^{13}$ - which is what we are working with.

Overall, the aim was to use these tools on longread data. Unfortunately the reads which were decent quality (plus good BLAST results) were short - so we couldn't see what more the tools potentially had to offer for this data.

We can see that from the research, some of the tools work very well with this new nanopore data. We can use various features to make observations; coming to understand how nanopore quality is quite low, even in good data: however this may just be average quality score for nanopore data, some papers have Phred score of $10.53[8]-1$ in 10 error (see supplementary material: current version figure 1).

We realise that the minION is a very sensitive technology and running the DNA through the nanopore needs to be a steady procedure; otherwise results are affected greatly: the $\mathrm{T}$ count was very unusually high.

Many state of the art bioinformatics tools are not yet suitable for long-read data.

We take away from this research with an idea of bioinformatics tools and how well they work with this new long-read sequence data. Perhaps we can acknowledge the early state of nanopore sequencing itself - it is still biologically difficult to handle DNA and keep it intact for sequencing.

\section{Acknowledgements}

I would like to thank Amanda Clare (afc@aber.ac.uk), Senior Lecturer at the Institute of IMPACS (Institute of Mathematics, Physics and Computer Science), my supervisor throughout this project for the constant guidance and support.

I would also like to acknowledge, Andre Soares (ans74@aber.ac.uk), PhD student in Geomicrobiology at IBERS (Institute of Biological, Environmental $\mathscr{E}$ Rural Sciences), who was a research member a part of the team who went in the mine - he assisted me throughout and provided information about the datasets whenever I asked.

Finally, I want to express appreciation for Samuel M Nicholls (msn@aber.ac.uk), PhD student of Bioinformatics at IBERS and the Department of Computer Science, who creator of Goldilocks - he helped with installation of some tools.

\section{References}

[1] Simon Andrews. A quality control tool for high throughput sequence data. 2010, 2015.

[2] NCBI Resource Coordinators. Database resources of the national center for biotechnology information. Nucleic acids research, 44(Database issue):D7, 2016.

[3] Susana OL Direito, Andries Marees, and Wilfred FM Röling. Sensitive life detection strategies for low-biomass environments: optimizing extraction of nucleic acids adsorbing to terrestrial and mars analogue minerals. FEMS microbiology ecology, 81(1):111-123, 2012.

[4] Arwyn Edwards, Andre Soares, Sara Rassner, Paul Green, Joao Felix, and Andrew Mitchell. Deep sequencing: Intra-terrestrial metagenomics illustrates the potential of off-grid nanopore dna sequencing. bioRxiv, 2017.

[5] Daehwan Kim, Li Song, Florian P Breitwieser, and Steven L Salzberg. Centrifuge: rapid and sensitive classification of metagenomic sequences. Genome research, 26(12):1721-1729, 2016.

[6] Heng Li, Bob Handsaker, Alec Wysoker, Tim Fennell, Jue Ruan, Nils Homer, Gabor Marth, Goncalo Abecasis, and Richard Durbin. The sequence alignment/map format and samtools. Bioinformatics, 25(16):2078-2079, 2009.

[7] Nicholas J Loman and Aaron R Quinlan. Poretools: a toolkit for analyzing nanopore sequence data. Bioinformatics, 30(23):3399-3401, 2014.

[8] Samuel M Nicholls, Wayne Aubrey, Arwyn Edwards, Kurt de Grave, Sharon Huws, Leander Schietgat, André Soares, Christopher J Creevey, and Amanda Clare. Computational haplotype recovery and long-read validation identifies novel isoforms of industrially relevant enzymes from natural microbial communities. bioRxiv, 2017.

[9] Samuel M Nicholls, Amanda Clare, and Joshua C Randall. Goldilocks: a tool for identifying genomic regions that are 'just right'. Bioinformatics, 32(13):2047-2049, 2016.

\footnotetext{
${ }^{13}$ Centrifuge, "Classifier for metagenomic sequences". https://github.com/infphilo/centrifuge
} 